\title{
Development
}

\section{Process evaluation of infertility management in primary care: has open access HSG been normalized?}

\author{
Scott Wilkes ${ }^{1}$ and Greg Rubin ${ }^{2}$ \\ ${ }^{1}$ Institute of Health and Society, Newcastle University, Newcastle upon Tyne, UK \\ ${ }^{2}$ Durham University School of Medicine and Health, Wolfson Research Institute, Stockton on Tees, UK
}

\begin{abstract}
Aim: To map the results of four empirical quantitative and qualitative studies to the Normalization Process Model (NPM) to explain why open access hysterosalpingography (HSG) for the initial management of infertile couples has or has not normalized in primary care. Background: The NPM is an applied theoretical model to help understand the factors that lead to the routine embedding of a complex intervention in everyday practice. Open access HSG has recently become available for the initial assessment of infertility in primary care. Methods: The results of two qualitative studies (a focus group study and an in-depth interview study with patients and professionals) and two quantitative studies (a pilot survey and a pragmatic clusterrandomized controlled trial) evaluating open access HSG are interpreted by mapping the results to the NPM. Findings: Application of the model shows that open access HSG would confer an advantage to all agencies if they could be sure that the expertise was present and supported within primary care. Conclusions: Open access HSG was adopted but not normalized into everyday practice. Despite demonstration of modest workability, it has been counteracted by limited integration. Further evaluation of integration within contexts is required.
\end{abstract}

Key words: family practice; hysterosalpingography; infertility; normalization; open access investigations; primary care

Received 13 February 2009; accepted 22 June 2009; first published online 28 July 2009

\section{Introduction}

On average, general practitioners (GPs) see one or two infertile couples each year (Wilkes and Jones, 1995). The recommended initial investigations of semen analysis, endocrine blood tests and tubal patency testing (National Institute for Health and Clinical Excellence, 2004) are performed by some

Correspondence to: Scott Wilkes, Institute of Health and Society, Newcastle University, 21 Claremont Place, Newcastle upon Tyne, NE2 4AA, UK. Email: scott.wilkes@newcastle. ac.uk

(C) 2009 Cambridge University Press
GPs (Wilkes and Jones, 1995; Das and Chin, 2003; Nicopoullos and Croucher, 2003; Morrison et al., 2007). The initial management of infertility in primary care is influenced by a number of components. These include GPs' confidence and competence, their perceived responsibilities, open access to diagnostic tests and access to specialist services (Wilkes et al., 2007). Open access to tubal patency testing with hysterosalpingography (HSG) as part of infertility assessment in primary care, is part of a complex intervention influenced by the behaviour of practitioners and the methods of organizing and delivering those behaviours (Medical Research 
Council, 2000; Wilkes et al., 2007). Evaluating complex interventions has been framed recently as a two-stage process using both quantitative and qualitative methods in each stage (Campbell et al., 2007): first, a process to define and understand the problem, and develop and understand the intervention, and then to optimize the evaluation. This process describes the context of the problem and includes health service systems, characteristics of the population, the prevalence and severity of the condition and also its location and change over time.

Theories (Grol et al., 2007) are applied to conceptually understand and describe the pathway by which a complex intervention may attain its goals. The process involves mapping out the mechanisms and pathways believed to lead from the intervention to the desired outcomes, then adding evidence and data to this map (Campbell et al., 2007). The outcome will be one of three scenarios: the intervention is unlikely to be cost-effective or work; the intervention is obviously beneficial and should be implemented immediately; or there is a state of equipoise and the intervention requires evaluation in a definitive trial. The Normalization Process Model (NPM) is an applied theoretical model that uses four constructs to predict or evaluate the uptake of a complex intervention such that it becomes routinely embedded (normalized) in everyday practice (May, 2006; May et al., 2007a). The NPM can be used to explain why open access HSG is used in one practice but not another. We mapped the results of four empirical quantitative and qualitative studies to the NPM to explain why open access HSG for the initial management of infertile couples has or has not normalized in primary care.

\section{Method}

A summary of the NPM and its constructs can be found in Figure 1. The NPM describes the interaction between the social processes of actors (patients, GPs and fertility specialists); objects, institutionally sanctioned means by which knowledge and practice are enacted (open access HSG and fertility guidelines) and contexts (the GP practice, primary care trust (PCT), GP contract) (May et al., 2007a). The NPM employs four constructs to explain the factors that affect the process of normalization: interactional workability, relational integration, skill-set

ENDOGENOUS (Professional) FACTORS

Interactional workability: Normalisation is likely if it confers an advantage to the actors. Congruence: Normalisation is likely if the actors have a shared belief in the Process.

Disposal: Normalisation is likely if the actors have a shared belief in the goals.

Relational Integration: Normalisation is likely if it fits the actors' role. Accountability: Normalisation is likely if actors have the necessary expertise. Confidence: Normalisation is likely if actors believe it falls within their remit.

EXOGENOUS (Organisational) FACTORS

Skill-set workability: Normalisation is likely if actors' skill-set requirements are agreed within contexts.

Allocation: Normalisation is likely if actors' responsibilities are agreed within contexts.

Performance: Normalisation is likely if the level to which actors perform is agreed within contexts.

Contextual integration: Normalisation is likely if it confers an advantage within contexts.

Execution: Normalisation is likely if resourcing issues are agreeable between/within contexts.

Realisation: Normalisation is likely if organisational systems between/within contexts are minimally disrupted.

Adapted from May 2007 bmc FP and BMC HSR

Figure 1 The Normalization Process Model: core constructs and propositions

Primary Health Care Research \& Development 2009; 10: 290-298 
workability and contextual integration (May et al., 2007b). In this paper, the results of four studies evaluating open access HSG in the initial management of infertility in general practice were mapped to the NPM constructs (Wilkes, 2007). A brief outline of each study is presented, and the studies themselves are described elsewhere (Wilkes et al., 2006; 2007; 2009a; 2009b).

\section{Pilot survey (Wilkes et al., 2006)}

Aim: To evaluate the uptake of open access HSG, speed of access to specialist services and the quality of the information recorded in the referral letter.

Design: Descriptive survey.

Setting: We made HSG available to six general practices in Newcastle upon Tyne, with a combined list size of 80500 patients, as an open access investigation.

Participants: Using hospital clinical records, we tracked the outcome of all infertile couples from the six pilot practices over a nine-month period.

\section{Focus group study (Wilkes et al., 2007)}

Aim: To explore GPs' perceptions of and attitudes to the initial management of the infertile couple, and their views on open access to HSG.

Design: Qualitative study using three focus groups. Setting: Seven general practices in Newcastle upon Tyne and Northumberland.

Subjects: We purposively selected three focus groups to provide a range of GPs' views. In total, 13 practitioners participated: 11 GPs, one GP registrar and one Nurse practitioner.

\section{Pragmatic cluster-randomized controlled trial (Wilkes et al., 2009a)}

Aim: To test whether providing GPs with open access to HSG results in infertile couples progressing to a diagnosis and management plan sooner than with usual management.

Design: A pragmatic cluster-randomized controlled trial.

Setting: 71 of 173 general practices in Northeast England agreed to participate.

Method: 670 infertile couples presented to 33 intervention practices and 25 control practices. Practices allocated to the intervention group had access to HSG for those infertile women who fulfilled pre-defined eligibility criteria. The primary outcome measure was the interval between presentation to the GP and the couple receiving a diagnosis and management plan.

\section{In-depth interview study (Wilkes et al., 2009b)}

Aim: To explore the perceptions and attitudes of patients and professionals to open access HSG for the initial management of the infertile couple in general practice.

Design: A nested qualitative study using in-depth interviews with GPs, fertility specialists and infertile couples.

Setting: Participants from Northumberland, Newcastle upon Tyne, North Tyneside, South Tyneside and Gateshead.

Participants: 39 interviewees: 12 GPs, 5 fertility specialists and 13 infertile couples (9 interviewed with their partner).

\section{Results}

The NPM constructs are described by a question relevant to the introduction of open access HSG, followed by an interpretation of the results from the four studies in the multi-method evaluation.

\section{Interactional workability}

If open access HSG is performed by GPs, will this confer an advantage to patients, GPs and fertility specialists, and do they have a shared belief in the process and goals of open access HSG?

The majority of patients and fertility specialists believed that open access HSG should be performed in general practice. Less than half of the GPs felt it was the role of individual GPs, though the majority of GPs felt it was appropriate for general practice to take on the role.

Personally I think it is a good thing. I think the way it was set up was very safe and sensible. It worked out very well because essentially it means that the patients that we receive have been fully investigated and really it is my job just to commence treatment then.

(Fertility specialist; interviewee S5) (Wilkes et al., 2009b)

I thought it was much better... a much quicker option just to go through the GP.

(Female patient; interviewee F10) (Wilkes et al., 2009b) 
I think there is a lot of things we now do. I don't see why we shouldn't be using that open access [HSG] as well as all the other [open access investigation] areas.

(GP; interviewee GP5) (Wilkes et al., 2009b)

In the cluster-randomized trial, $9 \%$ of infertile couples had open access HSG performed and reached the primary outcome sooner than those that did not (Wilkes et al., 2009a). The uptake of open access HSG could have risen to a maximum of $25 \%$ if all eligible couples were put forward for the investigation (Wilkes et al., 2006). In other words, approximately one-third to a half of those eligible had it organized by the GPs.

Patients generally felt that open access HSG would speed up the investigation process, although some felt it may delay things and just wanted onward referral. Specialists saw it as speeding up the process and allowing them to get on with the next stage of infertility management but GPs had conflicting views.

It's a hard one. As I say I don't see this being particularly useful but I would be quite happy to keep it, as theoretically it should streamline things for patients and it should make managing the couple easier for them. (GP; interviewee GP4) (Wilkes et al., 2009b)

I think it is useful because then I can present the information and make a decision whether we refer or not and I presume when they get there that is one of the first things that they are going to do, so, it is time aside for the hospital as well because when you do send them off you have got all the information they need.

(GP; interviewee GP11) (Wilkes et al., 2009b)

A minority of GPs could see that it would have benefit upon appropriate referral, speed up the whole patient journey and, in a limited number of cases, open up treatment options in general practice. Patients had a better-anticipated outcome of open access HSG than GPs, who believed that it would shape their future management. Fertility specialists were much more positive, believing that open access HSG would improve the quality of the initial investigation, subsequent referral and speed of access to diagnosis and treatment. Finally, infertility presents relatively infrequently to GPs so workload appeared not to be an issue, but there was an issue keeping up to date and having readily accessible and usable guidelines.

\section{Relational Integration}

Do GPs, fertility specialists and patients believe that GPs have the necessary expertise to request open access HSG, and does it fall within the remit of the GP?

I think primary care can organise the HSG. It's part of the first lot of investigations and it's the GP who sees the patients at the beginning and indeed throughout their lives. If they don't do it then someone else will have to do it. If it's appropriately done I don't think it matters who organises it.

(Fertility specialist; interviewee S1) (Wilkes et al., 2009b)

Well it is just another open access investigation available isn't it?

(GP; interviewee GP8) (Wilkes et al., 2009b)

Patients and fertility specialists saw open access HSG as a sensible service development and something that should happen. GPs, however, were unsure and concerned that patients should be seeing someone who deals with infertility frequently. For the basic steps of semen analysis, ovulatory blood tests and open access HSG, fertility specialists and patients perceived this role to be within the expertise of the GP.

I think all GP's should be able to do that [sperm tests, blood tests and open access HSG] for their patients.

(Female patient; interviewee F5) (Wilkes

et al., 2009b)

However, GPs felt that they saw infertile couples very infrequently, lacked expertise and had little opportunity to rehearse the necessary skills. Currently open access HSG is not described in authoritative guidelines. The interview studies suggest that open access HSG fits the role of a GP, perhaps a GP with an informal interest on behalf of a group of GPs, which was the experience of the interviewee below.

You could have within a partnership, somebody who sees them as a general rule; 
because most group practices will have certain GPs who dip into more specialist things.

[GP; interviewee GP2) (Wilkes et al., 2009b)

\section{Skill-set workability}

Do general practice, primary care commissioners, NHS guidelines and specialist services agree that the responsibility and skills for arranging $H S G$ rest with the GP?

Organizing blood tests and a semen analysis already happens and is recognized within the Royal College of Obstetricians and Gynaecologists guidelines (RCOG, 1999). Open access HSG is simply filling in another form as one specialist put it!

What expertise do you need? You need a pen and fill in a form.

(Fertility specialist; interviewee S2) (Wilkes et al., 2009b)

The difficulty may come at the next stage of interpretation and further management. Specialists and patients believe that all GPs should have the basic level of knowledge, that is, blood tests, semen analysis and open access HSG. GPs, however, were divided on their opinions, some believing that all GPs should get on and do it whilst others felt it should be a GP with an interest.

I think maybe one of the messages is that if we do decide that this will be a useful thing, perhaps rather than each of us see couples, one person in the practice takes it on.

[GP; Focus group A) (Wilkes et al., 2007)

Some patients also expressed a desire to go directly to the specialist without seeing the GP, although this was a minority view; the majority felt that the responsibility for the initial investigations lies with the GP.

GP first and then the specialist. (Female patient; interviewee F8) (Wilkes et al., 2009b)

Open access HSG had more proponents than opponents from each of the interviewee groups, but there is currently no infertility care pathway described for primary care and no support for open access HSG in general practice in current guidelines.

\section{Contextual integration}

Does open access HSG fit with the practices way of working, the PCT and the wider NHS and does it confer an advantage on these agencies?

Both the pilot survey and the cluster-randomized controlled trial demonstrated a shorter time for couples to reach a diagnosis and management plan for those that had open access HSG performed (Wilkes et al. 2006; Wilkes et al. 2009a). This was reflected in the opinion of the interviewees.

GPs should be able to do the HSG. It will relieve a lot of work in the IVF clinics

(Female patient; interviewee F6) (Wilkes

et al., 2009b)

We went to the clinic. The nurse who was in the place was doing a questionnaire and she was saying she was really really impressed with what Dr [GP] had done, she said all the tests were already done, and all the results were there and that saved her about 6 week's worth of work, so she was really impressed.

(Male patient; interviewee F6) (Wilkes et al., 2009b)

There are however organizational tensions. Open access HSG may identify women with tubal problems who require direct referral to a specialist in vitro fertilization (IVF) unit. This may threaten the current role for secondary care fertility services, which are becoming increasingly redundant, largely as a result of legislation restricting their activity in fertility treatments.

If a GP does an HSG and it says both tubes are blocked, the patient needs IVF. I, in secondary care do not do IVF, I don't provide that service, so for a GP to refer that patient to me, and for me to write a letter to the Centre for Life I think is wasteful.

(Fertility specialist; interviewee S3)

(Wilkes, 2007)

As a result, fertility management of the infertile couple is distilling out into two areas: first, the initial stages (semen analysis, endocrine blood tests and tubal patency tests) and second, the advanced stages, which occur predominantly in tertiary care. There are financial tensions. Open access HSG is a test that is done anyway on the 
patient journey, that is, there is no additional financial cost to the NHS. It is a test done in hospitals but organized by GPs. Despite being cost neutral to the NHS, there may be budgetary shifts between primary and secondary care to reflect the change in activity. This will undoubtedly lead to resourcing tensions between primary and secondary care. For example, the cost of HSG is currently within the general gynaecology budget. If general practice were charged for open access HSG then the general gynaecology budget would be reduced to reflect the shift in costs.

Although a relatively small workload, open access HSG and, more specifically, infertility management attract no GP Quality and Outcomes Framework payments, which has proved to be an effective tool to modify GP behaviour (Department of Health, 2009). This is best described by the theory of operant conditioning (Thorndike, 1901), which links behaviour and consequences. Open access HSG confers little advantage to general practice but potentially has greater value to commissioning agencies, fertility specialists, infertile couples and the wider NHS.

Mapping the results of the four studies to the NPM constructs shows that the qualitative studies informed all four constructs whilst the quantitative studies gave limited information to some of the constructs (Figure 2). A summary interpretation of the results of the four studies mapped to the NPM constructs is given in Figure 3.

\section{Discussion}

\section{Main findings}

The retrospective application of the NPM to the four studies has given a useful process evaluation of open access HSG. The model has largely been informed by the qualitative studies (Figure 2). Patients, GPs and fertility specialists felt that open access HSG would confer an advantage to all agencies if they could be sure that the expertise was present and supported within primary care. Performing open access HSG within primary care was supported and evidenced by the modest uptake of open access HSG in both the survey and cluster-randomized controlled trial. Couples that experienced open access HSG reached a diagnosis and management plan sooner than those with traditional management. However, a significant barrier

\begin{tabular}{|c|c|c|c|c|c|}
\hline Constructs & Propositions & $\begin{array}{c}\text { Focus } \\
\text { Group }\end{array}$ & $\begin{array}{c}\text { Pilot } \\
\text { Survey }\end{array}$ & CRCT & $\begin{array}{c}\text { Patient \& } \\
\text { Professional } \\
\text { interviews }\end{array}$ \\
\hline Interactional \\
workability & Congruence & Y & y & y & Y \\
\cline { 2 - 6 } & Disposal & Y & N & N & Y \\
\hline Relational & Accountability & Y & y & y & Y \\
\hline \hline
\end{tabular}

\begin{tabular}{|c|c|c|c|c|c|}
\hline integration & Confidence & Y & y & y & Y \\
\hline \hline $\begin{array}{c}\text { Skill-set } \\
\text { workability }\end{array}$ & Allocation & Y & N & N & Y \\
\cline { 2 - 6 } & Performance & Y & N & N & Y \\
\hline $\begin{array}{c}\text { Contextual } \\
\text { integration }\end{array}$ & Execution & N & N & N & y \\
\cline { 2 - 6 } & Realisation & $\mathrm{y}$ & $\mathrm{N}$ & $\mathrm{N}$ & $\mathrm{y}$ \\
\hline
\end{tabular}

Y: Informed the NPM construct $y$ : Minimally informed the NPM construct $\mathrm{N}$ : Did not inform the NPM construct

Figure 2 Mapping the results of the four empirical studies to inform the Normalization Process Model constructs

to its routine use was a general perception that infertility management is the responsibility of the fertility specialist. With the infrequent presentation of infertile couples and difficulty in keeping up to date with clinical management (Wilkes et al., 2007), it has assumed a low priority in general practice (Bowling, 1996). Infertility management is not part of the GP 'Quality and Outcomes Framework' contract (Department of Health, 2009) and open access HSG lacks support in local or national guidance (RCOG, 1999; NICE, 2004).

\section{Strengths and weaknesses}

None of the studies addressed the context of commissioning an open access HSG service within the NHS, nor did it address policy documents. Hence, the NPM construct of contextual integration was poorly informed, although the indepth interviews did provide a patient and health professional perspective on this issue.

\section{Comparison with other literature}

The NPM model has typically been used in qualitative studies (Finch, 2008; Gask et al., 2008; 


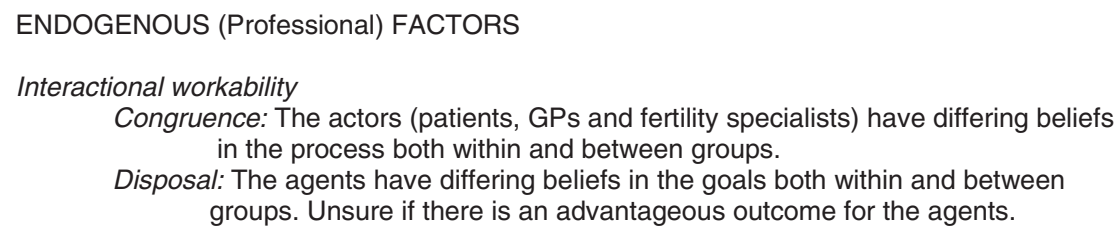

Relational Integration

Accountability: Actors believe GPs should perform open access HSG and have the necessary expertise to do it.

Confidence: Most actors believe it falls within the GP remit although this is new and not supported by authoritative sources of knowledge.

EXOGENOUS (Organisational) FACTORS
Skill-set workability
Allocation: Actors' responsibilities are not agreed within/between contexts with regard to open access HSG.
Performance: The level to which GPs should perform is not defined within/between contexts.

\section{Contextual integration}
Execution: This would require a change in policy and resource allocation within and between contexts.
Realisation: Organisational systems within and between contexts would have to change.

SUMMARY: Open access HSG for the initial management in primary care has been adopted but not normalised.

Figure 3 Normalization Process Model results for open access hysterosalpingography

Mair et al., 2008); however, in this project, a survey and cluster-randomized controlled trial have also contributed to the evaluation. The NPM has been applied to two complex trials: the delivery of problem solving therapies for psychosocial distress, and the delivery of nurse-led clinics for heart-failure treatment in primary care (May et al., 2007b). These evaluations have demonstrated adoption (not normalization) with problems relating to workability and integration in their immediate contexts. In evaluations of teledermatology (Finch, 2008) and telecare of chronic obstructive airways disease (Mair et al., 2008), their normalization was limited by the actors' belief in the process and goals (interactional workability); the former by the incongruence of the patients needs and the latter by the nurses' concerns over the safety and efficiency of the process. By contrast, clinical governance in primary mental health (Gask et al., 2008) and the use of decision support technologies in patientprofessional interactions (Elwyn et al., 2008) is limited by the context in which they operate (contextual integration), and it is this construct that is often under-researched and poorly understood.

\section{Implications for future practice}

In considering the likely normalization of open access HSG, consideration must be given to existing patterns of service organization, professional practice, patient-professional interaction, as well as clinical and cost-effectiveness (May et al., 2007b).

\section{Conclusion}

The 'active ingredient' that makes open access HSG work, may be difficult to define. Health 
services research often requires a mixed methods approach to gain in-depth insights into the process and outcomes, and ultimately the usefulness of an intervention. Broadly, experimental, well-controlled quantitative research will give information on what has happened within a research context, with specified measurable outcomes, such as time to appropriate treatment; qualitative research will give reasons why interventions have given the results we observe. Put simply, the two quantitative studies told us what happened; the two qualitative studies told us why it happened.

The NPM has given an explanation why open access HSG has been adopted but not normalized into everyday general practice. The challenge, now, is to define and address the constructs within the model that are limiting normalization and propose methods to address these barriers. Modeling a complex intervention and mapping the barriers is currently a process seldom undertaken in complex intervention trials (Bosch et al., 2007). The NPM has been a useful tool to describe the likely normalization of open access HSG for the initial management of infertility in primary care.

\section{Acknowledgements}

We would like to acknowledge the NHS National Coordinating Centre for Research Capacity Development, who funded this work through an award held by SW. Primary Care Researcher Development Award, National Co-ordinating Centre for Research Capacity Development, Blenheim House, Duncombe Street, Leeds, LS1 4PL. All researchers involved in the production of this paper are independent from the funder. Ref: RDA03/26.

We would also like to acknowledge the sponsor, Sunderland University, where this work was hosted. Academics within the university contributed to the study design; collection, analysis and interpretation of data; production of the paper and the decision to submit the article for publication. Ref: ID1746.

The authorship contributions are as follows: SW and GR designed and implemented the study. SW carried out the data collection. SW wrote the paper. SW and GR contributed to the data analysis and the editing of the paper. SW and GR act as guarantors of the study and accept full responsibility for the conduct of the study, data access and controlled the decision to publish. Carl May, who commented on drafts of this paper, is duly acknowledged.

Ethical approval for this study was granted from Newcastle and North Tyneside as the main REC with SSA approval from Northumberland, Gateshead and South Tyneside Local Research Ethics Committees. Ref: 04/Q0901/33; 2003/201. Approval was also granted fro Sunderland University research ethics committee.

There are no competing interests to declare.

\section{References}

Bosch, M., van der Weijden, T., Wensing, M. and Grol, R. 2007: Tailoring quality improvement interventions to identified barriers: a multiple case analysis. Journal of Evaluation in Clinical Practice 13, 161-68.

Bowling, A. 1996: Health care rationing: the public's debate. British Medical Journal 312, 670-74.

Campbell, N.C., Murray, E., Darbyshire, J., Emery, J., Farmer, A., Griffiths, F., Guthrie, B., Lester, H., Wilson, P. and Kinmonth, A.L. 2007: Designing and evaluating complex interventions to improve health care. British Medical Journal 334, 455-59.

Das, S. and Chin, K.A. 2003: A study of pre-referral evaluation of infertile couples. Journal of Obstetrics and Gynaecology 23, 70 .

Department of Health. 2009: Quality and outcomes framework. National GP contract. Retrieved 22 January 2009 from http://www.dh.gov.uk.

Elwyn, G., Legare, F., van der Weijden, T., Edwards, A. and May, C. 2008: Arduous implementation: does the Normalisation Process Model explain why it's so difficult to embed decision support technologies for patients in routine clinical practice. Implementation Science 31, 57, doi:10.1186/1748-5908-3-57.

Finch, T. 2008: Teledermatology for chronic disease management: coherence and normalization. Chronic Illness 4, 127-34.

Gask, L., Rogers, A., Campbell, S. and Sheaff, R. 2008: Beyond the limits of clinical governance? The case of mental health in English primary care. BMC Health Services Research 26, 63.

Grol, R.P., Bosch, M.C., Hulscher, M.E., Eccles, M.P. and Wensing, M. 2007: Planning and studying improvement in patient care: the use of theoretical perspectives. Milbank Quarterly 85, 93-138.

Mair, F.S., Hiscock, J. and Beaton, S.C. 2008: Understanding factors that inhibit or promote the utilization of telecare in chronic lung disease. Chronic Illness 4, 110-17.

May, C. 2006: A rational model for assessing and evaluating complex interventions in health care. BMC Health Services Research 6, 86. 
May, C., Finch, T., Mair, F., Ballini, L., Dowrick, C., Eccles, M., Gask, L., MacFarlane, A., Murray, E., Rapley, T., Rogers, A., Treweek, S., Wallace, P., Anderson, G., Burns, J. and Heaven, B. 2007a: Understanding the implementation of complex interventions in health care: the normalization process model. BMC Health Services Research 7, 148.

May, C.R., Mair, F.S., Dowrick, C.F. and Finch, T.L. 2007b: Process evaluation for complex interventions in primary care: understanding trials using the Normalisation Process Model. BMC Family Practice 8, 42.

Medical Research Council. 2000: A framework for developement and evaluation of RCTs for complex interventions to improve health. London: Medical Research Council.

Morrison, C., Bhattacharya, S., Hamilton, M., Templeton, A. and Smith, B. 2007: Initial management of infertility: an audit of pre-referral investigations and exploration of couples' views at the interface of primary and secondary care. Human Fertility 10, 25-31.

National Institute for Health and Clinical Excellence. 2004: Fertility: assessment and treatment for people with fertility problems. London: National Institute for Clinical Excellence, 11.

Nicopoullos, J.D. and Croucher, C.A. 2003: Audit of primary care and initial secondary care investigations set against RCOG guidelines as standard in cases of sub fertility. Journal of Obstetrics and Gynaecology 23, 397-401.

Royal College of Obstetricians and Gynaecologists. 1999: Evidence-based clinical guidelines. Guideline summary no.
2: The initial investigation and management of the infertile couple. BJU International JID - 100886721 83, 636-40.

Thorndike, E.L. 1901: Animal intelligence: an experimental study of the associative processes in animals. Psychological Review 2, 1-109.

Wilkes, S. 2007: Evaluation of open access HSG in the initial management of infertility in primary care, $\mathrm{PhD}$ thesis. University of Sunderland.

Wilkes, S. and Jones, K. 1995: Retrospective review of the prevalence and management of infertility in women in one practice over a five year period. British Journal of General Practice 45, 75-77.

Wilkes, S., Murdoch, A., Rubin, G., Chinn, D. and Wilsdon, J. 2006: Investigation of infertility management in primary care with open access hysterosalpingography (HSG): a pilot study. Human Fertility 9, 47-51.

Wilkes, S., Hall, N., Crosland, A., Murdoch, A. and Rubin, G. 2007: General practitioners' perceptions and attitudes to infertility management in primary care: focus group study. Journal of Evaluation in Clinical Practice 13, 358-63.

Wilkes, S., Murdoch, A., Steen, N., Wilsdon, J. and Rubin, G. 2009a: Open Access Tubal assessment for the initial management of infertility in general practice (the OATS trial): a pragmatic cluster randomised controlled trial. British Journal of General Practice 59, 329-35.

Wilkes, S., Rubin, G., Crosland, A., Hall, N. and Murdoch, A. 2009b: Patient and professional views of open access Hysterosalpingography (HSG) for the initial management of infertility in primary care. British Journal of General Practice 59, 336-42. 